\title{
POEMS Syndrome Presenting with Acute Demyelinating Polyneuropathy: Increased Terminal Latency Indices and Uniform Demyelination
}

\author{
Ju-Hong Min ${ }^{1}$, Dong Kyu Lee ${ }^{1}$, Sujin Lee ${ }^{1}$, Kihyun Kim², Eun-Seok Jeon ${ }^{3}$, \\ Hye Ryoun Jang ${ }^{4}$ and Byoung Joon Kim ${ }^{1}$
}

\begin{abstract}
We herein report a case of polyneuropathy, organomegaly, endocrinopathy, M-protein and skin changes (POEMS) syndrome presenting with acute Guillain-Barré syndrome (GBS)-like features. The patient was healthy, except for mild dilated cardiomyopathy. She was unable to walk within ten days of the onset of weakness. A nerve conduction study (NCS) showed length-dependent, symmetric and non-focal demyelinating features with increased terminal latency indices (TLIs) in the lower limbs. Following the administration of intravenous immunoglobulin infusion, the proximal weakness of the lower extremities improved; however, the pleural effusion was aggravated and ascites newly developed. On further work-ups, splenomegaly, Mprotein and sclerotic bone changes were observed. This case suggests that, although rare, POEMS syndrome can present with acute demyelinating polyneuropathy resembling GBS and that characteristic NCS features such as increased TLI and uniform demyelination are helpful for the early diagnosis of POEMS syndrome.
\end{abstract}

Key words: POEMS syndrome, Guillain-Barré syndrome, nerve conduction study, polyneuropathy

(Intern Med 52: 1513-1516, 2013)

(DOI: 10.2169/internalmedicine.52.9335)

\section{Introduction}

Polyneuropathy, organomegaly, endocrinopathy, M-protein and skin changes (POEMS) syndrome is a rare cause of polyneuropathy (1). However, the presence of polyneuropathy is essential for the diagnosis of POEMS and is the most common initial manifestation of the syndrome. Usually, the polyneuropathy of this disease is characterized by chronic and progressive demyelinating features, which are emphasized in the differentiation of POEMS from chronic inflammatory demyelinating polyneuropathy (CIDP) (2). We herein report the case of a patient with POEMS syndrome who presented with an acute progressive polyneuropathy resembling Guillain-Barré syndrome (GBS) (3).

\section{Case Report}

A 63-year-old woman visited our hospital due to a gait disturbance. Fourteen days previously, she felt a tingling sensation in the distal limbs, and 10 days previously she developed acute weakness of the lower limbs. There were no other symptoms such as diplopia, dysarthria, dysphagia, dizziness or urinary or defecation difficulties. Her past medical history included dyspnea, sweating and pitting edema six months previously, which were considered to be symptoms of dilated cardiomyopathy and were being controlled with diuretics. In addition, she had been suffering from diabetes and hypertension for seven years. She exhibited predominant lower extremity weakness; the motor power in the upper extremities was MRC grade 4+, while the motor power in the

\footnotetext{
${ }^{1}$ Department of Neurology, Samsung Medical Center, Sungkyunkwan University School of Medicine, Korea, ${ }^{2}$ Department of Oncology, Samsung Medical Center, Sungkyunkwan University School of Medicine, Korea, ${ }^{3}$ Department of Cardiology, Samsung Medical Center, Sungkyunkwan University School of Medicine, Korea and ${ }^{4}$ Department of Nephrology, Samsung Medical Center, Sungkyunkwan University School of Medicine, Korea

Received for publication November 14, 2012; Accepted for publication January 7, 2013

Correspondence to Dr. Byoung Joon Kim, bjkim@skku.edu
} 
Table 1. Nerve Conduction Studies Performed 14 Days after Symptom Onset

\begin{tabular}{|c|c|c|c|c|}
\hline Motor (stimulation site) & $\begin{array}{l}\text { Distal latency } \\
\text { (ms) (Normal } \\
\text { limit) }\end{array}$ & $\begin{array}{l}\text { CMAP amplitude, } \\
\text { distal/proximal (mV) } \\
\text { (Normal limit) }\end{array}$ & $\begin{array}{c}\text { Conduction } \\
\text { elocity (m/s) } \\
\text { (Normal limit) }\end{array}$ & $\begin{array}{l}\text { F-wave latency } \\
\text { (ms) (Normal } \\
\text { limit) }\end{array}$ \\
\hline Median, L (wrist, elbow) & $4.6(3.6)$ & $8.49 / 6.63(5)$ & $39(49.96)$ & $38.8(26.65)$ \\
\hline Median, R (wrist, elbow) & $4.7(3.6)$ & $7.15 / 6.37(5)$ & 37 (49.96) & $41.1(26.65)$ \\
\hline Ulnar, L (wrist, elbow) & $3.1(2.51)$ & $12.57 / 9.92(5)$ & $43(50.61)$ & $41.5(26.62)$ \\
\hline Ulnar, R (wrist, elbow) & $2.7(2.51)$ & $11.56 / 9.15(5)$ & $39(50.61)$ & $40.9(26.62)$ \\
\hline $\begin{array}{l}\text { Peroneal, L (ankle, fibular } \\
\text { head) }\end{array}$ & $5.0(4.78)$ & $1.13 / 0.64(4)$ & $26(41.85)$ & NP (44.93) \\
\hline $\begin{array}{l}\text { Peroneal, R (ankle, fibular } \\
\text { head) }\end{array}$ & $4.9(4.78)$ & $1.43 / 0.78(4)$ & $27(41.85)$ & NP (44.93) \\
\hline $\begin{array}{l}\text { Tibial, L (ankle, popliteal } \\
\text { fossa) }\end{array}$ & $5.9(5.11)$ & $8.29 / 4.17(5)$ & $27(40.63)$ & $54.2(47.35)$ \\
\hline $\begin{array}{l}\text { Tibial, R (ankle, popliteal } \\
\text { fossa) }\end{array}$ & $5.9(5.11)$ & $8.14 / 4.00(5)$ & $29(40.63)$ & $55.0(47.35)$ \\
\hline Sensory (stimulation site) & & $\begin{array}{l}\text { SNAP amplitude }(\mu \mathrm{V}) \\
\text { (Normal limit) }\end{array}$ & $\begin{array}{c}\text { Conduction } \\
\text { velocity }(\mathrm{m} / \mathbf{s}) \\
\text { (Normal limit) }\end{array}$ & \\
\hline Median, L (wrist) & & $6.65(10)$ & $39(41.26)$ & \\
\hline Median, R (wrist) & & $6.01(10)$ & $38(41.26)$ & \\
\hline Ulnar, L (wrist) & & $9.36(8)$ & $43(39.26)$ & \\
\hline Ulnar, R (wrist) & & $13.7(8)$ & $41(39.26)$ & \\
\hline Sural, L (calf) & & $1.37(6)$ & $34(34.6)$ & \\
\hline Sural, R (calf) & & $3.67(6)$ & $34(34.6)$ & \\
\hline Superficial peroneal, L (calf) & & NP (4) & NP (40.5) & \\
\hline Superficial Peroneal, R (calf) & & NP (4) & NP (40.5) & \\
\hline
\end{tabular}

lower extremities was MRC grade 3 or 4 and both hip flexors in particular showed severe weakness of MRC grade 1. The sensations of touch, pain and vibration were decreased by $80 \%$ in the distal part of the extremities, although the position sense was intact. The patient's deep tendon reflexes were decreased; the biceps, triceps, knee and ankle jerks were areflexic and no pathologic reflexes were observed. The cerebellar function test and the Romberg test showed no abnormalities. A nerve conduction study (NCS) revealed symmetric demyelinating polyneuropathy (Table 1). The spinal fluid demonstrated mild pleocytosis (WBC: $4 / \mu \mathrm{L}$ ) with a markedly elevated protein level $(114.5 \mathrm{mg} / \mathrm{dL}$, normal range: $20-40 \mathrm{mg} / \mathrm{dL}$ ). Blood test results for anti-GM1 antibodies (IgG) were negative. The patient was diagnosed with GBS and treated with intravenous immunoglobulin at a dose of $400 \mathrm{mg} / \mathrm{kg}$ for five days. Two days after treatment, the weakness improved; the hip flexors achieved an MRC grade of 3 and the motor power of the other muscles was similar. However, the patient's weight increased by $2 \mathrm{~kg}$, and the dyspnea and pitting edema were aggravated. Moreover, abdominal distension newly developed. On further work-ups, protein electrophoresis and serum immunofixation revealed monoclonal gammopathy (M-peak in gamma globulin region) and abnormal bands against anti-IgA and anti-lambda. A bone marrow biopsy disclosed plasma cell myeloma (IgA, lambda). Pelvic bone CT showed sclerotic changes, and abdominal ultrasonography revealed splenomegaly and ascites with a pleural effusion. Chest X-ray showed cardiomegaly and a pleural effusion, while echocardiography revealed di- lated cardiomyopathy with moderate to severe left ventricular systolic dysfunction. No definitive skin changes were observed, and no endocrinopathies except diabetes were detected. Finally, the patient was diagnosed with POEMS syndrome according to the relevant criteria (1). We calculated the terminal latency indices (TLIs) in this patient using the following formula: TLI=terminal distance $(\mathrm{mm}) /($ distal motor latency $(\mathrm{ms}) \times$ motor nerve conduction velocity $(\mathrm{m} /$ s)) (4). The values were 0.28 and 0.29 (median), 0.38 and 0.47 (ulnar), 0.62 and 0.60 (peroneal) and 0.63 and 0.58 (tibial) in the left and right sides. To evaluate the clinical usage of TLI in the diagnosis of POEMS syndrome, we acquired TLIs in three groups consisting of 15 healthy persons (58.00 \pm 14.39 years; range: $42-77$ years; female: male $=8: 7$ ), 15 GBS patients (61.48 \pm 7.62 years; range: $47-71$ years; female:male $=6: 9)$ and 15 CIDP patients $(61.66 \pm 6.63$ years; range: $47-71$ years; female:male $=7: 8$ ) who visited our hospital (Table 2). In the median and ulnar nerves, our patient exhibited similar or higher TLIs than the mean TLIs of the healthy persons and GBS and CIDP patients. However, the TLI values of our patient were within the ranges of TLIs measured in the GBS and CIDP groups. In contrast, in the peroneal and posterior tibial nerves, the TLIs of our patient were higher than the mean TLIs of the three groups and were also beyond the upper limits of the TLIs of these groups.

Two weeks after treatment with immunoglobulin, the patient's weakness worsened to MRC grade 3 in the upper extremities and MRC grade $0-1$ in the lower extremities. 
Table 2. Terminal Latency Indices in this POEMS Patient, Healthy Subjects $(n=15)$, GBS Patients $(n=15)$ and CIDP Patients $(\mathbf{n}=15)$

\begin{tabular}{lcccc}
\hline Nerve & $\begin{array}{c}\text { This POEMS } \\
\text { patient }(\mathbf{R} / \mathbf{L})\end{array}$ & $\begin{array}{c}\text { Healthy subjects } \\
\text { (range), } \mathbf{n = 1 5}\end{array}$ & $\begin{array}{c}\text { GBS (range), } \\
\mathbf{n}=\mathbf{1 5}\end{array}$ & $\begin{array}{c}\text { CIDP (range), } \\
\mathbf{n}=\mathbf{1 5}\end{array}$ \\
\hline Median & $0.28 / 0.29$ & $0.29 \pm 0.03$ & $0.18 \pm 0.08$ & $0.28 \pm 0.11$ \\
& & $(0.25-0.33)$ & $(0.06-0.31)$ & $(0.05-0.45)$ \\
Ulnar & \multirow{2}{*}{$0.47 / 0.38$} & $0.36 \pm 0.62$ & $0.28 \pm 0.09$ & $0.34 \pm 0.08$ \\
& & $(0.33-0.40)$ & $(0.18-0.49)$ & $(0.16-0.49)$ \\
Peroneal & $0.62 / 0.60$ & $0.40 \pm 0.04$ & $0.32 \pm 0.09$ & $0.38 \pm 0.10$ \\
& & $(0.33-0.47)$ & $(0.14-0.43)$ & $(0.12-0.51)$ \\
Tibial & $0.63 / 0.58$ & $0.51 \pm 0.04$ & $0.39 \pm 0.10$ & $0.45 \pm 0.10$ \\
& & $(0.44-0.57)$ & $(0.23-0.57)$ & $(0.25-0.57)$ \\
\hline
\end{tabular}

POEMS: polyneuropathy, organomegaly, endocrinopathy, $\mathrm{M}$ protein, and skin changes, GBS: Guillain-Barre syndrome, CIDP: chronic inflammatory demyelinating polyneuropathy, $\mathrm{N}$ : number, $\mathrm{R}$ : right, $\mathrm{L}$ : left

Therefore, she decided to receive treatment with two cycles of cyclophosphamide and prednisolone, followed by another two cycles of cyclophosphamide and dexamethasone. After chemotherapy, her motor power improved to MRC grade 4 (upper extremities) and MRC grade 2-3 (lower extremities).

\section{Discussion}

Our patient was initially diagnosed with GBS because her weakness was acute and rapidly aggravated (3). She was unable to walk within 14 days, an interval that is quite shorter than that observed in other POEMS syndrome patients (5). Usually, the neuropathy of this syndrome exhibits a chronic progressive course characterized by sensorimotor symptoms $(1,6)$. Recently, Isose et al. reported that 22 of 30 patients with POEMS syndrome were unable to walk independently at the first visit, with a median duration from disease onset of 9.5 months (5). Among these patients, one presented with acute-onset neuropathy 10 days after developing diarrhea and experienced rapid progression within 14 days (5). In contrast, our patient reported no preceding events such as diarrhea or upper respiratory infections before the onset of neuropathy. Instead, she had suffered from dyspnea, sweating, peripheral edema and a pleural effusion caused by underlying dilated cardiomyopathy. In patients with POEMS syndrome, organomegaly usually consists of hepatomegaly, splenomegaly and/or lymphadenopathy; however, cardiomegaly has also been suggested to be a feature of POEMS syndrome (7). Interestingly, the present patient's motor power improved after the infusion of immunoglobulin, which initially led to the diagnosis of GBS. Previously, it has been hypothesized that immunoglobulin treatment may benefit POEMS patients by downgrading the vascular endothelial growth factor (VEGF) release induced by inflammatory cytokines (8). However, the effectiveness of immunoglobulin related to this mechanism was unclear in our patient because the serum VEGF level was not assessed. Moreover, the patient's motor power, which initially improved, worsened within two weeks.
Electrophysiologically, differentiating between POEMS syndrome and CIDP can be difficult since the neuropathy of POEMS syndrome exhibits chronic demyelinating features. However, as described in Table 1, the NCS features observed in our patient did not show laterality, focal/patch demyelinating features such as definite conduction block or abnormal temporal dispersion or diverse degrees of abnormalities between nerves regarding conduction velocities and distal latencies. This length-dependent, symmetric and uniform demyelinating neuropathy without laterality could have been due to intoxication or POEMS syndrome, rather than GBS or CIDP.

Moreover, the increase in TLI as well as the overproduction of VEGF is known to be helpful for making the differential diagnosis of POEMS syndrome (9-11). In a previous report of 12 Korean POEMS syndrome patients, the TLIs were $0.32 \pm 0.06$ (median), $0.45 \pm 0.07$ (ulnar), $0.59 \pm 0.03$ (tibial) and $0.58 \pm 0.83$ (peroneal) (9). In our patient, the TLIs of both peroneal nerves $(0.62$ and 0.60 ), both tibial nerves (0.63 and 0.58$)$ and the right ulnar nerve $(0.47)$ were greater than the mean values, as previously reported, although those of both median nerves (0.28 and 0.29) and the left ulnar nerve $(0.38)$ were not greater than previously reported values. Cocito et al. (12) measured TLIs in typical CIDP patients, as follows: 0.34-0.40 (median), 0.41-0.47 (ulnar), 0.34-0.38 (peroneal) and 0.40-0.48 (tibial). Compared to the values observed in these CIDP patients, the TLIs of our patient were also much higher in the lower extremities. Moreover, the peroneal and tibial TLIs of our patient were much higher than those of our GBS and CIDP patients as well as the healthy controls, as observed in Table 2 . We suggest that POEMS syndrome patients can present with acute progressive neuropathy and can be responsive to immunoglobulin. However, the characteristic NCS findings, such as increased TLIs, particularly in the lower limbs, with length-dependent, symmetric and uniform demyelination can be helpful for making an early diagnosis of POEMS syndrome.

The authors state that they have no Conflict of Interest (COI).

\section{References}

1. Dispenzieri A. POEMS syndrome. Blood Rev 21: 285-299, 2007.

2. Van den Bergh PY, Hadden RD, Bouche P, et al. European Federation of Neurological Societies/Peripheral Nerve Society guideline on management of chronic inflammatory demyelinating polyradiculoneuropathy: report of a joint task force of the european federation of neurological societies and the peripheral nerve society-first revision. Eur J Neurol 17: 356-363, 2010.

3. Yuki N, Hartung HP. Guillain-Barré syndrome. N Eng J Med 366: 2294-2304, 2012.

4. Shahani BT, Young RR, Potts F, Maccabee P. Terminal latency index (TLI) and rate responsive studies in motor neuron disease (MND), peripheral neuropathies, and entrapment syndromes. Acta Neurol Scand 73 (suppl 60): 118, 1979.

5. Isose $\mathrm{S}$, Misawa $\mathrm{S}$, Kanai $\mathrm{K}$, et al. POEMS syndrome with Guillan-Barré syndrome-like acute onset: a case report and review of neurological progression in 30 cases. J Neurol Neurosurg Psy- 
chiatry 82: 678-680, 2011.

6. Mathis S, Magy L, Kabore R, Faugeras F, Richard L, Vallat JM. POEMS syndrome with prominent acute axonal lesions. J Neurol Sci 313: 185-188, 2012.

7. Tanus T, Miller HJ. POEMS syndrome presenting with cardiomegaly and cardiomyopathy. J Intern Med 231: 445-448, 1992.

8. Terracciano $\mathrm{C}$, Fiore $\mathrm{S}$, Doldo $\mathrm{E}$, et al. Inverse correlation between VEGF and soluble VEGF receptor 2 in POEMS with AIDP responsive to intravenous immunoglobulin. Muscle Nerve 42: 445 448, 2010.

9. Min JH, Hong YH, Lee KW. Electrophysiological features of patients with POEMS syndrome. Clin Neurophysiol 116: 965-968,
2005.

10. Sung JY, Kuwabara S, Ogawara K, Kanai K, Hattori T. Patterns of nerve conduction abnormalities in POEMS syndrome. Muscle Nerve 26: 189-193, 2002.

11. Nasu S, Misawa S, Sekiguchi Y, et al. Different neurological and physiological profiles in poems syndrome and chronic inflammatory demyelinating polyneuropathy. J Neurol Neurosurg Psychiatry 83: 476-479, 2012

12. Cocito $D$, Isoardo $G$, Ciaramitaro $P$, et al. Terminal latency index in polyneuropathy with $\operatorname{IgM}$ paraproteinemia and anti-MAG antibody. Muscle Nerve 24: 1278-1282, 2001.

(C) 2013 The Japanese Society of Internal Medicine http://www.naika.or.jp/imonline/index.html 